\title{
AC Losses of Roebel Cables with Striated 2G REBCO Strands
}

\author{
Yifeng Yang ${ }^{(1)}$, Jorge Pelegrin ${ }^{(1)}$, Edward A. Young ${ }^{(1)}$, Rainer Nast, Anna Kario, and Wilfried Goldacker
}

\begin{abstract}
We present experimental data on the ac losses in Roebel cables with striated 2 G REBCO strands. Electromagnetic measurements by pick-up coils were carried out at different temperatures between $10 \mathrm{~K}$ and $85 \mathrm{~K}$ at frequencies between $2 \mathrm{~Hz}$ and $20 \mathrm{kHz}$ in transverse ac fields up to $0.5 \mathrm{~T}$. The Roebel cable samples were assembled from laser cut $2 \mathrm{G}$ REBCO strands, which consist of striated filaments prepared with laser scribing. Measurements at different temperatures show that the Roebel cables with striated strands have a lower losses by a factor of strand filament number over the whole temperature range. For analysis, a semiquantitative approach for unstriated Roebel was found to work satisfactorily. However, the Striated Roebel cables appears to have a different field penetration from those of the unstriated Roebel. The coupling current among the filaments has a time constant shorter than $0.1 \mathrm{~ms}$ and do not appear to increase in the striated Roebel cables, hence insignificant for most magnets related applications.
\end{abstract}

Index Terms-REBCO $2 \mathrm{G}$ conductor, straition, Roebel cable, ac losses.

\section{INTRODUCTION}

$\mathbf{H}$ IGH current cables are essential for some superconducting devices such as accelerator magnets, superconducting magnetic storage coils and superconducting fault current limiters. Such cables are required to consist of several transposed superconducting strands to ensure uniform current distribution for field quality and cryogenic stability. While the sustained research and development of $2 \mathrm{G} \mathrm{REBCO}$ superconducting tapes have led to impressive critical current performances in high magnetic fields, the flat geometry of these tape conductors poses significant challenges to the assembling of fully transposed cables. One of the few possibilities is that of a Roebel bar [1]-[4] which was originally invented more than a hundred years ago for reducing the eddy current losses in high current cables/bus-bars for conventional machines.

Manuscript received September 21, 2017; accepted February 9, 2018. Date of publication February 16, 2018; date of current version March 20, 2018. This work was supported by EuCARD-2. EuCARD-2 is co-funded by the partners and the European Commission under Capacities seven Framework Programme under Grant 312453. (Corresponding author: Yifeng Yang.)

Y. Yang, J. Pelegrin, and E. A. Young are with the Institute of Cryogenics, Faculty of Engineering and the Environment, University of Southampton, Southampton SO17 1BJ, U.K. (e-mail: y.yang@soton.ac.uk; jpm1c13@ soton.ac.uk; E.A.Young@ soton.ac.uk).

R. Nast, A. Kario, and W. Goldacker are with the Karlsruhe Institute of Technology, Karlsruhe 76131, Germany (e-mail: rainer.nast@kit.edu; anna.kario@ kit.edu; wilfried.Goldacker@kit.edu).

Color versions of one or more of the figures in this paper are available online at http://ieeexplore.ieee.org.

Digital Object Identifier 10.1109/TASC.2018.2807178

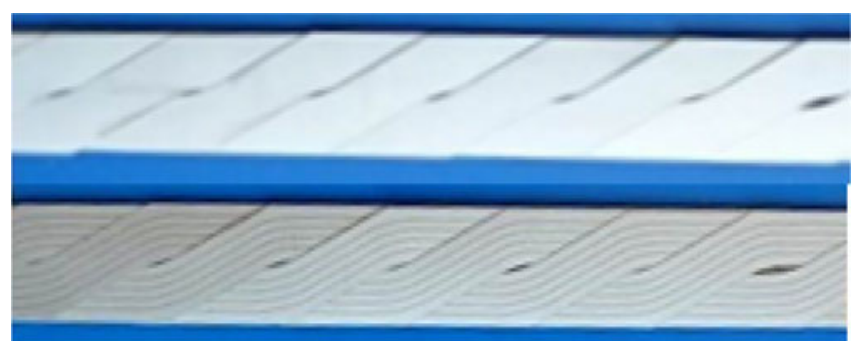

Fig. 1. Pictures of Roebel samples with the top and bottom for cables of unstriated strands and 5-filament strands respectively.

With the growing availability of $2 \mathrm{G}$ REBCO tapes in long length, several laboratories [2], [3] have been producing prototype Roebel cables with punched or laser-cut strands for the important studies on the critical current performance and manufacturing optimization. In addition, there have also been studies [5]-[8] on the magnetization and ac losses, which inform on both the effectiveness of the Roebel transposition in homogenizing the current distribution and the complex magnetic interactions among the strands.

The hysteresis loss in superconductors is most effectively reduced by subdivision into fine filaments. In addition these filaments must be twisted at a sufficiently short pitch in order to ensure that they are magnetically uncoupled under desired ac conditions. Although striated parallel filaments can be made successfully by laser scribing to reduce ac losses in short samples [9], it is topologically challenging to twist them for uncoupling over long length. The strand transposition provides a longitudinally alternating magnetic disposition, which might be an effective block [6] against the long length coupling of the striated filaments. Following on a previous promising report [10] on reduced losses in Roebel cable with striated strands, the present work reports systematic loss measurements on cable samples of different striated filaments over an extended temperature, field and frequency range. The results, together with data from single striated strands and standard Roebel cable, confirm that striated filaments are indeed uncoupled in Roebel cables with further loss reduction from that found in an earlier study [10].

\section{METHODS}

\section{A. Samples}

The cable samples (Fig. 1) were made using SP-M3-675/SPKIT-20090521-1 SuperPower $12 \mathrm{~mm}$ wide Ag tape. The tapes were cut with laser to $5.5 \mathrm{~mm}$ width and Roebel shape. 
Filaments were formed by laser scribing subsequently. Finally the tapes were placed in a furnace for $1 \mathrm{~h}\left(\mathrm{O}_{2}\right.$ atmosphere, $500{ }^{\circ} \mathrm{C}$ ) in order to oxidize the laser scribed tracks for an increased resistivity across the groove and the tape. The Roebel cables have a $126 \mathrm{~mm}$ transposition pitch and are assembled using 10 strands into $100 \mathrm{~mm}$ long samples. Three samples of unstriated strands, $5(1 \mathrm{~mm})$ and $10(0.5 \mathrm{~mm})$ filaments per Roebel strand were studied.

\section{B. Measurements}

The ac loss measurement setup allows ac magnetic fields up to $0.2 \mathrm{~T}$ and sample temperature down to $10 \mathrm{~K}$ by contact cooling. The superconductor magnetization are measured at $5 \mathrm{~Hz}$ where the coupling among filaments can be considered negligible. The coupling development were studied by measurement at higher frequencies.

\section{A SEMI-QuANTITATIVE APPROACH FOR LOSSES IN "STANDARD" ROEBEL CABLES}

We attempt to a semi-quantitative analysis of the ac losses of Roebel cables based on the applied field losses of a thin strip [11]. For a strip of width $w$, cross-section area $S$, line critical current density $\sigma_{c}=I_{c} / w$, and hysteretic loss per unit length $Q$, the peak of the loss factor $\Gamma(\beta)=Q /\left(2 \mu_{0} H_{0}^{2} S\right)$ is not at $H_{c}=$ $\sigma_{c} / w\left(c f\right.$. [11]) but almost $150 \%$ higher at $H_{P}=2.46421 H_{c} \sim$ $2.5 \times 69 / 70 H_{c}$, where an effective full penetration or saturation of hysteresis can be reached. For convenience, the loss factor may be adapted approximately as

$$
\Gamma=\frac{\pi}{5} \frac{w}{d} \beta^{-1}\left(\frac{4}{5 \beta} \ln \left(\cosh \left(\frac{5 \beta}{2}\right)\right)-\tanh \left(\frac{5 \beta}{2}\right)\right)
$$

where $\beta=H_{0} / H_{P}$ with the field amplitude $H_{0}$ normalized by $H_{P} \sim 5 \sigma_{c} / 2 \pi=5 / 2 H_{c}$ at a small error less than $1.5 \%$. The loss factor is dimensionless and useful for comparing finite thickness $(d)$ conductors of different critical current and nominal cross-section $S=w d$.

\section{A. Two Side-by-Side Strands}

A previous study [8] showed that, in a perpendicular ac magnetic field, two electrically isolated Roebel strands placed closely side-by-side are coupled magnetically through the compression of magnetic flux in between. The corresponding ac loss $Q_{2 \mathrm{MC}}$ is $40 \%$ more than the losses $Q_{2 \mathrm{UC}}=2 Q_{s}$ of two "truly" isolated strands placed far apart. The subscript "MC" refers to magnetically coupled, "UC" to uncoupled, and "s" for a single strand. Connecting two side-by-side strands electrically results in full coupling over long length, i.e. the two strands become a single strand of twice the width with the corresponding ac loss $Q_{2 \mathrm{FC}}=2 Q_{2 \mathrm{UC}}$. The fully coupled (FC) and uncoupled (UC) configurations have the same saturation field $H_{P}$ as that of a single strand as the critical current per unit width is unchanged. Despite a much increased flux density between the side-by-side strands, the saturation field for the magnetically coupled was found only about $25 \%$ lower. Hence approximately for all ac field amplitude $H_{0}$,

$$
\begin{aligned}
Q_{2 \mathrm{FC}}\left(H_{0}\right) & \sim \sqrt{2} Q_{2 \mathrm{MC}}\left(H_{0}\right) \\
& =2 Q_{2 \mathrm{UC}}\left(H_{0}\right)=4 Q_{s}\left(H_{0}\right)
\end{aligned}
$$

and

$$
\begin{aligned}
H_{P, 2 \mathrm{MC}} & \sim H_{P, 2 \mathrm{FC}}=H_{P, 2 \mathrm{UC}}=H_{P s} \\
& =\frac{5 I_{c s}}{2 \pi w_{s}}=\frac{5}{2 \pi} \sigma_{s}
\end{aligned}
$$

where $I_{c s}$ is the critical current of a single strand of width $w_{s}$ and $\sigma_{s}$ is the corresponding strand critical current per unit width. Note that a magnetically coupled strand has a lower loss than that of fully coupled strand. The corresponding loss factors satisfy $\Gamma_{2 \mathrm{MC}}=\Gamma_{2 \mathrm{FC}} / \sqrt{2}=\sqrt{2} \Gamma_{2 \mathrm{UC}}=\sqrt{2} \Gamma_{s}$. The magnetic coupling factor of $\sqrt{2}$ might change according to the distance between and the thickness of the side-by-side tape conductors, however, it can only vary within $[1,2)$ between fully uncoupled and coupled limits.

\section{B. Stacked Strands}

Roebel cables consist of two side-by-side bundles of stacked REBCO strands, which are transposed one by one between the sides. The number of stacked strands in each bundle in HTS Roebel cables is relatively small, as necessary for their suitability for coil winding. Thus such a relatively thin stack of strands tends to behave similar to a thin strip in perpendicular fields. The stack of $m$ strands has an increase line critical current $m \sigma_{c s}$ although the corresponding penetration field has been found [8] a fraction lower at

$$
H_{P m} \sim \frac{m}{1.6} H_{P s}
$$

possibly due to a decreased demagnetization for the stacks. The corresponding ac loss factor of stacked strands is

$$
\Gamma_{m s}(\beta)=m^{-1} \Gamma_{s}\left(\frac{1.6}{m} \beta_{s}\right)
$$

which, compared to a single strand, has a reduced peak loss factor to a fraction of $m$, albeit at a higher saturation field of $\frac{m}{1.6} H_{P s}$. The reduction factor of 1.6 for the stack penetration field is dependent on the stacks retaining a sufficient high aspect ratio, a feature necessary for winding applications nonetheless. To establish a more precise relation between the stack geometry and penetration field would be a highly relevant investigation for future.

\section{Roebel Cable as Two Magnetically Coupled Stacks of Tape Strands}

Considering a Roebel cable of $2 m$ strands as two magnetically coupled stacks of $m$ strands gives an estimated loss factor of

$$
\Gamma_{R 2 m}(\beta) \sim \frac{\sqrt{2}}{m} \Gamma_{s}\left(\frac{1.6}{m} \beta_{s}\right)
$$

which, as shown in Fig. 2, appears to work quite well for the measured loss factor of a 15-strand $(m=7.5, m / 1.6=$ 


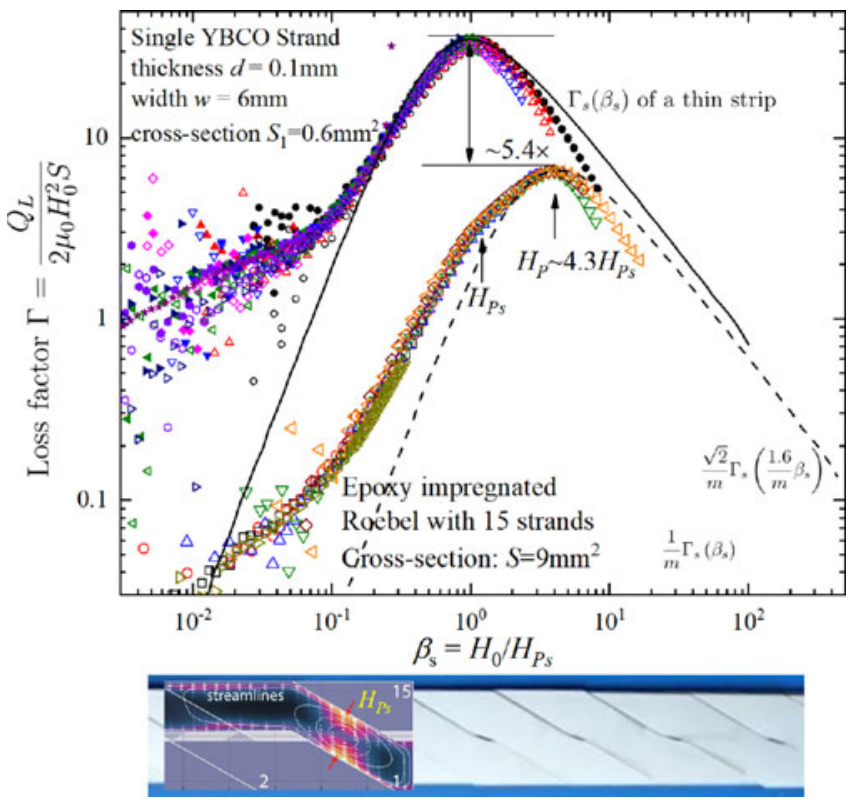

Fig. 2. Loss factor of a 15 REBCO strand Robel cable measured between $5 \mathrm{~K}$ and $90 \mathrm{~K}$ as a function of normalized field $\beta_{s}$ with reference to the strand saturation field $H_{P s}$. The dashed line corresponds to the prediction by the semi-quantitative calculation, as outlined in the text, for the 15-strand Roebel cable. The prominent kink in the loss factor at $\beta_{s} \sim 1$ suggests the full saturation of a strand undergoing transposition by the saturation field $H_{P s}$ of a single strand instead $H_{P m}$ of the stack, as illustrated conceptually by the lower graph.

$4.68, \sqrt{2} / m=1 / 5.3)$ Roebel, with the shift of the full saturation position and peak loss factor matching reasonably close to the predictions.

It should be noted that, although the peak loss factor of a $2 m$-strand Roebel cable is lower by a factor of $\sqrt{2} / m$ than the peak value for a single strand or $2 m$ uncoupled strands, the corresponding loss at the same field is not so much reduced. In fact the loss factor of a Roebel cable becomes comparable to that of uncoupled tapes at fields much higher than $H_{P m}$.

The pronounced kink found in the Roebel cable at $\beta_{s} \sim 1$ is attributable to the full penetration of a transposing strand, coinciding with the full penetration field $H_{P s}$ of a single strand rather than $H_{P m}$ of the stack (see lower image of Fig. 2). Although the $2 \mathrm{D}$ semi-quantitative approach does not consider transposition, its agreement with experimental results beyond single strand full penetration as well as the appearance of a kink at $H_{P s}$ suggest that the 3D aspects of transposition are largely confined to the transposing section of a single strand which alternates at a spatial wavelength of $T_{P} / 2 m$ from one strand to another along the transposition pitch $T_{P}$. Therefore the corresponding magnetization loss of transposition is equivalent to that of two long single strands at the top and bottom of the cable.

The 2D approach of side-by-side magnetic coupling of two stacks of $m$ tapes is within the scope of [12], however, a calculation based on a thin strip [11] is more straightforward and avoids the need for computer modelling.

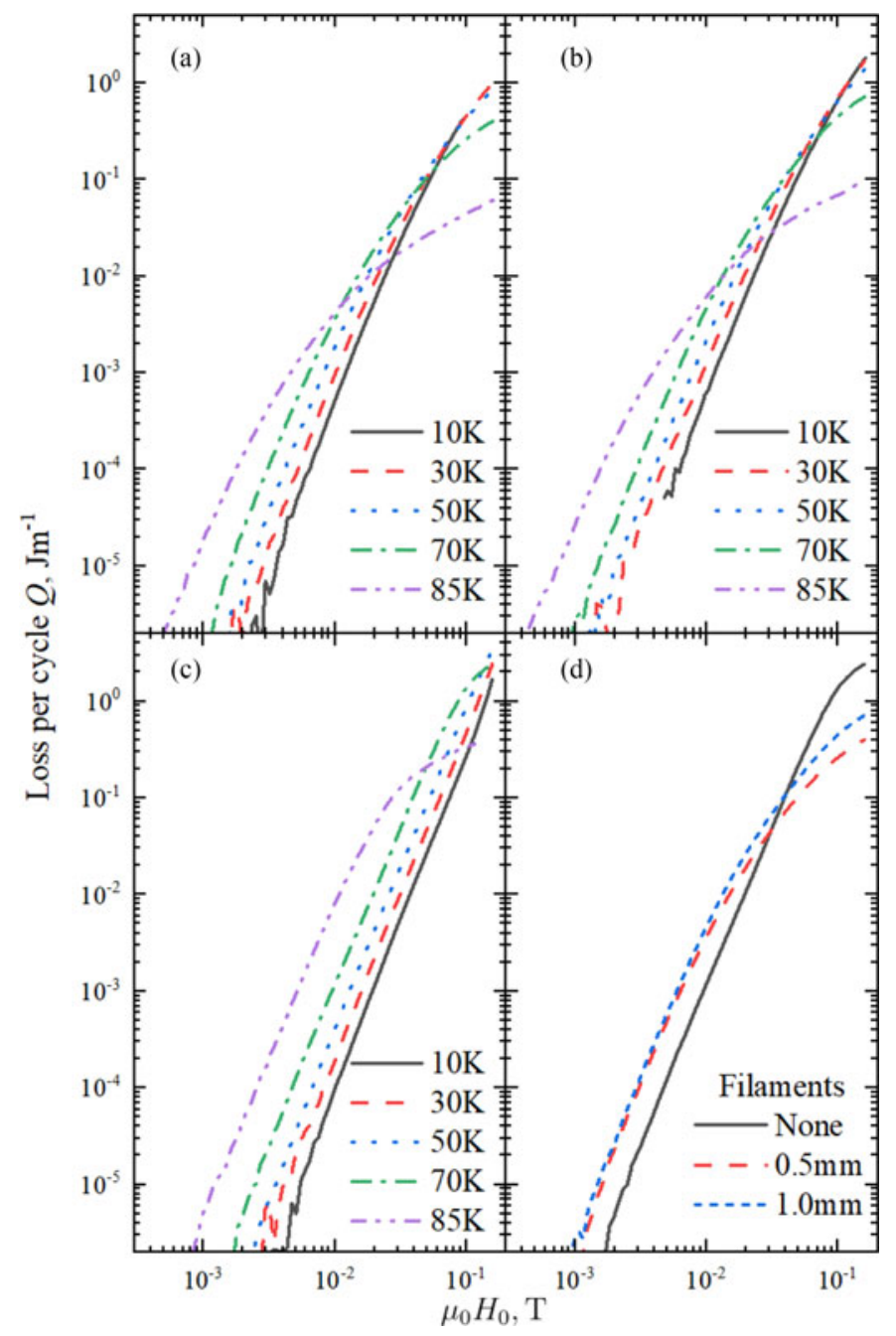

Fig. 3. Loss per unit length as a function of ac field amplitude $H_{0}$ measured at temperatures between $10 \mathrm{~K}$ and $85 \mathrm{~K}$ for Roebel cables with strands of (a) $0.5 \mathrm{~mm}$ filaments, (b) $1.0 \mathrm{~mm}$ filaments, and (c) no filaments. The losses at $75 \mathrm{~K}$ of three cables with different type strands shown together in (d) as a function of $H_{0}$.

\section{RESUlTS AND DISCUSSION}

\section{A. Reduction of Roebel Cable Magnetization Losses with Striated Strands}

The ac loss per cycle per unit length of the three Roebel samples of $0.5 \mathrm{~mm}$ filaments, $1.0 \mathrm{~mm}$ filaments, and unstriated strands was measured at $5 \mathrm{~Hz}$ and at different temperatures between $10 \mathrm{~K}$ and $85 \mathrm{~K}$, as shown in Fig. 3(a)/(b)/(c) respectively as a function of the field amplitude $H_{0}$. All the curves at sufficiently high temperatures exhibit a distinct change in the gradient in log-log scale, marking the full penetration of the magnetic field. As the critical current density increases with reducing temperature, the full penetration field $H_{P}$ increases accordingly with the $Q\left(H_{0}\right)$ curves moving towards higher fields. $Q\left(H_{0}\right)$ also becomes broader with striation, as shown in Fig. 3(d), and the losses are significantly lower than those of the unstriated beyond the full penetration. 


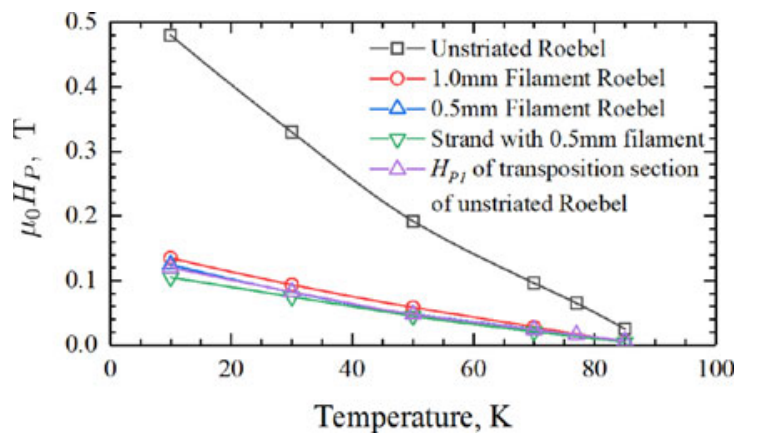

Fig. 4. Full saturation field $H_{P}$ of the Roebel samples as a function of temperature. $H_{P}$ of a single strand with $0.5 \mathrm{~mm}$ filaments is also shown (green inverted triangle).

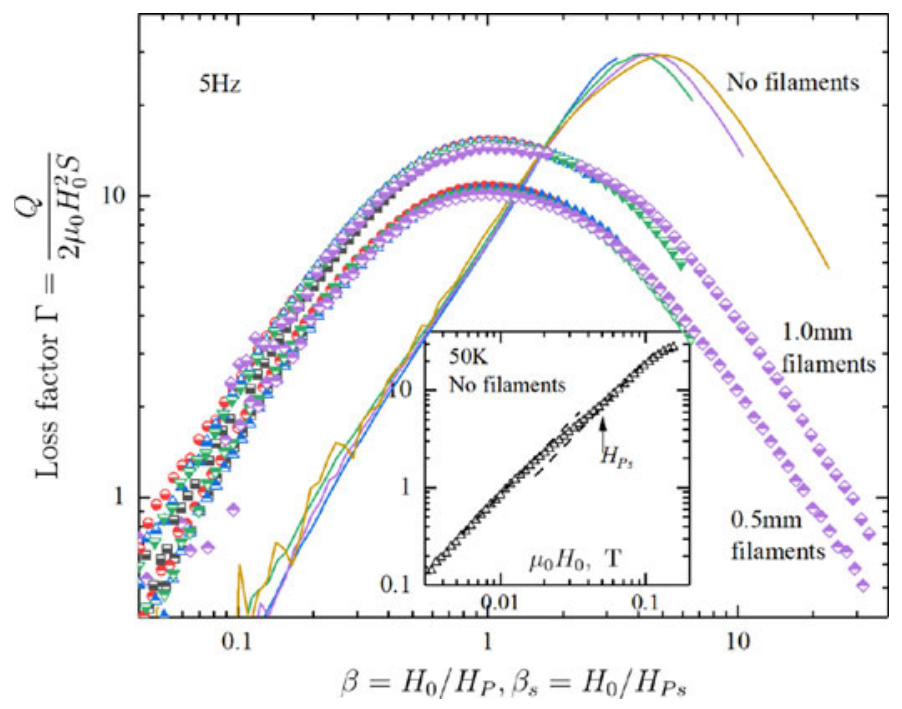

Fig. 5. Loss factor at different temperatures $(10 \mathrm{~K}-85 \mathrm{~K})$ as a function of normalized field $\beta$ for the Roebel samples with striated strands of $0.5 \mathrm{~mm}$ (upper filled symbols) and $1.0 \mathrm{~mm}$ (lower filled symbols) filaments. The loss factor for the Roebel sample with unstriated strands is plotted against a nominal $\beta_{s}$ of the strand where transposing strand is fully penetrated (see inset as an example).

The full penetration field of the Roebel samples at different temperatures can be determined from the peaks found in the loss factor $\Gamma=Q /\left(2 \mu_{0} H_{0}^{2} S\right)$, where $S=2 m w d, m=5, w=$ $5.5 \mathrm{~mm}, d=50 \mu \mathrm{m}$. As shown in Fig. 4, the full saturation field of the unstriated Roebel cable is about 4 times of those for the striated strands (green inverted triangle). The significantly lower saturation field of the striated strands is not due to a reduction of the critical current by laser scribing and is somewhat more than that expected [12] by the flux compression between the filaments. Although the saturation field $H_{P s}$ of the transposing section in the unstriated Roebel is quite subtle for a smaller strand number of 10 , it is still visible on closer inspection, as shown by the example of $50 \mathrm{~K}$ in the inset of Fig. 5. In contrast to the stacks in unstriated Roebe, the penetration field $H_{P}$ of the striated Roebel samples is only marginally higher than that of the single striated strand, i.e., the stacking of striated strands does not seem to increase the saturation significantly irrespective of the filament size.
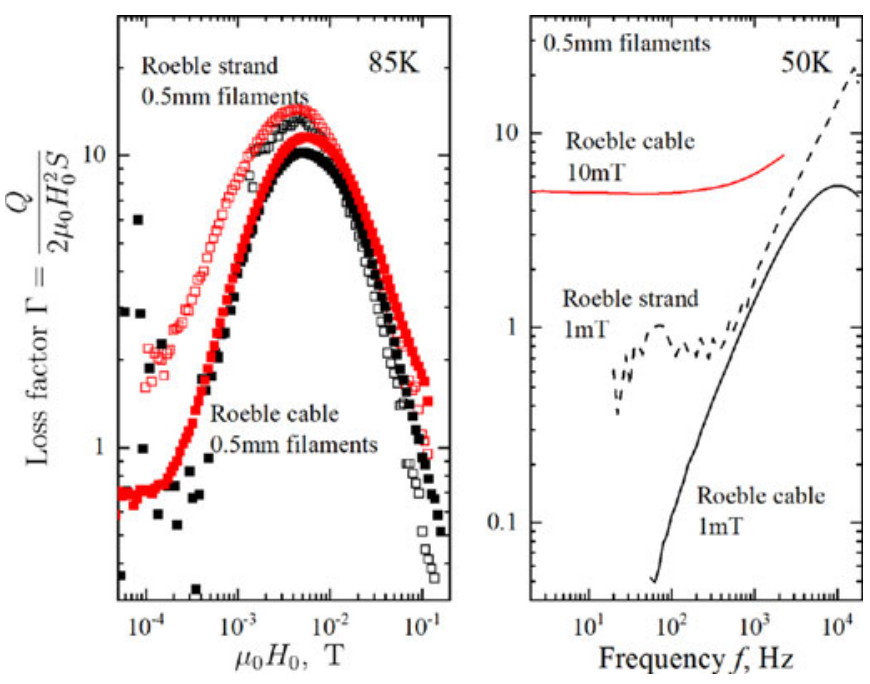

Fig. 6. Left: Loss factors of striated Roebel strand and cables as a function of field amplitude $H_{0}$ at $5 \mathrm{~Hz}$ (black symbols) and $59 \mathrm{~Hz}$ (red symbols) at $85 \mathrm{~K}$. Right: Loss factors of striated Roebel strand and cables as a function of frequency at $50 \mathrm{~K}$.

The main graph of Fig. 5 shows the loss factor as a function of the normalized field $\beta=H_{0} / H_{P}$ for the striated Roebels. Although their $\Gamma(\beta)$ is significantly broadened around the saturation field, there still remains a universal scaling by $H_{P}$ for different temperature. The loss factor for the $0.5 \mathrm{~mm}$-filament Roebel (upper filled symbols) is lower than that of the $1.0 \mathrm{~mm}$ filament one by a factor of 1.5 and 2 before and after the full saturation respectively.

The loss factor of the unstriated Roebel is shown in solid lines as a function of $\beta_{s}=H_{0} / H_{P s}$ for comparison. Since $H_{P s}$ of the unstriated is almost the same as the $H_{P}$ of the striated, the normalized field $\beta_{s}$ for the striated Roebel in Fig. 5 represents nearly the same field scale as $\beta$ for the striated. It becomes immediately clear that a saturated Roebel of unstriated strands has much greater losses than those of the striated by almost a factor given the number of filaments, i.e, approximately 5 and 10 times of the respective losses of Roebel cables with $1.0 \mathrm{~mm}$ and $0.5 \mathrm{~mm}$ filaments.

\section{B. Coupling Current}

Although some broadening of $\Gamma(\beta)$ for striated REBCO strands may be attributable [13] to the contribution of the coupling currents among the filaments, it does not appear to be the case for our samples. Shown on the left of Fig. 6 are the loss factors of a striated strand with $0.5 \mathrm{~mm}$ filaments (open symbols) and the corresponding Roebel cable (filled symbols) as a function of field amplitude $H_{0}$. Although the losses are higher at $59 \mathrm{~Hz}$ (red symbols) than at $5 \mathrm{~Hz}$ (black symbols) for both the strand and Roebel cable, the functional form of hysteresis $\Gamma\left(H_{0}\right)$ is preserved, except at very low fields less than $2 \mathrm{mT}$ where the loss factor becomes independent of $H_{0}$ as the magnetization losses becomes lower than the coupling current loss. The right pane of Fig. 6 shows the loss factors of a striated strand (dashed line) and striated cable (solid lines) as a function of frequency up to $20 \mathrm{kHz}$. The coupling current 
losses at $1 \mathrm{mT}$ dominate in the Robel cable (black line) for the measurable range above $50 \mathrm{~Hz}$ but is overtaken at $10 \mathrm{mT}$ by the magnetization loss at frequencies below $500 \mathrm{~Hz}$. The time constant of the coupling current appears to be around $0.1 \mathrm{~ms}$, due to a high inter-filament resistance attributed to the improved oxidation process after laser scribing.

\section{CONCLUSION}

Magnetization loss of Roebel cables with striated strands is reduced approximately by a factor of the number of filaments when fully penetrated. The coupling current losses is low in high field in frequencies up to a few hundred Hz. While a semiquantitative approach seems to work with unstriated Roebel cables, a quantitative understanding of losses in the striated Roebel cable is yet to be developed.

\section{REFERENCES}

[1] M. Leghissa, V. Hussennether, and H. W. Neumüller, "ka-class highcurrent HTS conductors and windings for large scale applications," Adv. Sci. Technol., vol. 47, pp. 212-219, Oct. 2006.

[2] N. J. Long, R. A. Badcock, K. Hamilton, A. Wright, Z. Jiang, and L. S. Lakshmi, "A treatise on electricity and magnetism development of YBCO roebel cables for high current transport and low ac loss applications," $J$. Phys., Conf. Series, 2010, vol. 234, Art no. 022021.
[3] W. Goldacker et al., "Status of high transport current ROEBEL assembled coated conductor cables," Supercond. Sci. Technol, vol. 22, 2009, Art. no. 034003.

[4] A. Badel et al., "REBCO cable for the EuCARD2 demonstrator magnet," IEEE Trans. Appl. Supercond, vol. 26, no. 3, Apr. 2016, Art. no. 4803908

[5] L. S. Lakshmi, K. P. Thakur, M. P. Staines, R. A. Badcock, and N. J. Long, "Magnetic AC loss characteristics of $2 \mathrm{G}$ Roebel cable," IEEE Trans. Appl. Supercond, vol. 19, no. 3, pp. 3361-3364, Jun. 2009.

[6] S. Terzieva et al., "Transport and magnetization ac losses of ROEBEL assembled coated conductor cables: Measurements and calculations," Supercond. Sci. Technol, vol. 23, 2010, Art. no. 014023.

[7] Z. Jiang et al., "Total AC loss measurements in a six strand Roebel cable carrying an AC current in an AC magnetic field," Supercond. Sci. Technol, vol. 26, 2013, Art. no. 035014.

[8] Y. Yang et al., "Magnetization losses of Roebel cable samples with $2 \mathrm{G}$ YBCO coated conductor strands," IEEE Trans. Appl. Supercond., vol. 26, no. 3, Apr. 2016, Art. no. 8202105.

[9] N. Amemiya et al., "AC loss reduction of YBCO coated conductors by multifilamentary structure," Supercond. Sci. Technol, vol. 17, pp. 14641471, 2004.

[10] S. Terzieva et al., "Investigation of the effect of striated strands on the AC losses of 2G Roebel cables," Supercond. Sci. Technol, vol. 24, 2014, Art. no. 045001

[11] E. H. Brandt and M. Indenbom, "Type-II-superconductor strip with current in a perpendicular magnetic field," Phys. Rev. B, vol. 48, pp. 12893-12906, 1993.

[12] E. Pardo, A. Sanchez, and C. Navau, "Magnetic properties of arrays of superconducting strips in a perpendicular field," Phys. Rev. B, vol. 67, 2003, Art. no. 104517

[13] E. Demencik et al., "Ac magnetization loss and transverse resistivity of striated YBCO coated conductors," IEEE Trans. Appl. Supercond, vol. 25, no. 3, Jun. 2015, Art. no. 8201405. 